\title{
Acute Cardiac Complications and Subclinical Myocardial Injuries Associated with Pheochromocytoma and Paraganglioma
}

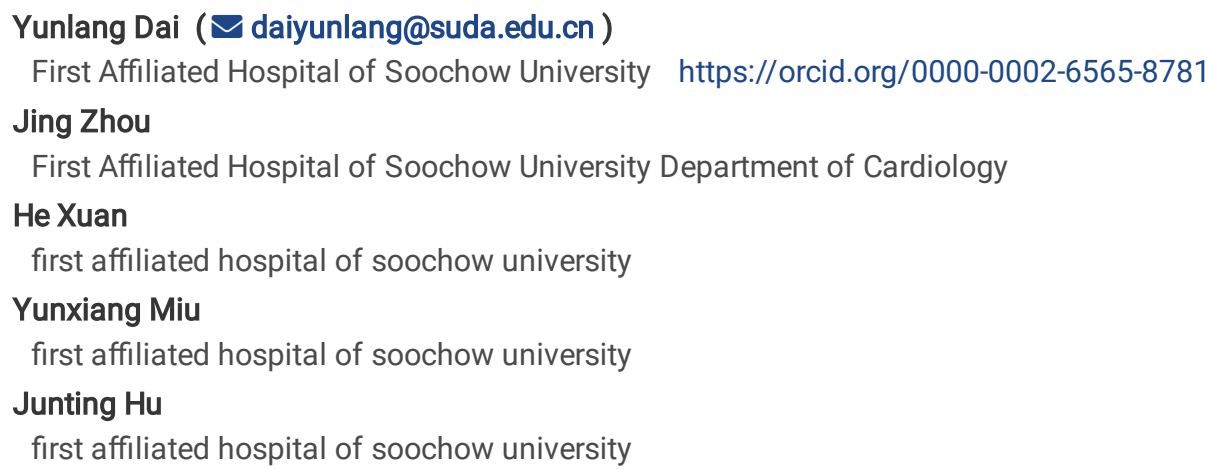

\section{Research article}

Keywords: pheochromocytoma, cardiac complication, myocardial injury, longitudinal strain

Posted Date: December 29th, 2020

DOI: https://doi.org/10.21203/rs.3.rs-134946/v1

License: (c) (i) This work is licensed under a Creative Commons Attribution 4.0 International License. Read Full License

Version of Record: A version of this preprint was published at BMC Cardiovascular Disorders on April 21st, 2021. See the published version at https://doi.org/10.1186/s12872-021-02013-6. 


\section{Abstract}

Background: Catecholamine excess arising from pheochromocytomas and paragangliomas (PPGLs) can cause a wide spectrum of cardiac manifestations, including acute cardiac complications (ACCs) and subclinical myocardial injuries (SMIs). Hence, we aimed to conduct a comprehensive analysis of ACCs and SMls in a large cohort of PPGLs.

Methods: We retrospectively analyzed the clinical data of consecutive patients with PPGLs admitted between January 2013 and July 2020 ( $n=$ 189). The prevalence and presentation of ACCs and SMIs were investigated, and comparisons were conducted between cases with and without ACCs.

Results: Fourteen patients (7.4\%) fulfilled the criteria for ACCs, consisting of nine cases (4.8\%) with Takotsubo-like cardiomyopathy, three cases $(1.6 \%)$ with heart failure with preserved ejection fraction, and the remaining one $(0.5 \%)$ with catecholamine-induced cardiomyopathy. Compared to those without ACCs $(n=175)$, patients with ACCs had higher prevalence of epinephrine-producing PPGLs $(81.8 \%$ vs $33.9 \%$. P $=0.006)$, and were more likely to show invasive behavior $(61.5 \%$ vs $27.3 \%, \mathrm{P}=0.022)$ and hemorrhage/necrosis $(53.9 \%$ vs $17.4 \%, \mathrm{P}=0.005)$ at histology. Among patients suffered Takotsubo-like cardiomyopathy, an apical sparing pattern $(5 / 7,71.4 \%)$ dominated in the impaired patterns of longitudinal strain (LS). In the end, a fairly high percent $(21 / 77,27.2 \%)$ of patients (excluding 14 cases with ACCs) who underwent screening troponin, natriuretic peptide, and echocardiography had SMls.

Conclusions: One in every fourteen PPGLs patients presented with ACCs; and, in the cases with Takotsubo-like cardiomyopathy, an apical sparing pattern was the primary pattern in LS. Furthermore, nearly one-third of patients without symptoms had SMls. The diagnosis of PPGLs should be considered in patients with acute reversible cardiomyopathy, especially with an apical sparing pattern in LS.

\section{Background}

Pheochromocytomas (PHEOs) and paragangliomas (PGLs) (PPGLs) are rare tumors, secreting catecholamines (CAs), and have a wide variety of cardiovascular manifestations and complications, which were reviewed recently[1]. Clinically, the presentations of PPGLs are usually unspecific, and only a limited proportion of patients display classic triad of headache, palpitation and sweating, resulting in the delay in diagnosis, especially in cases with cardiac complications[2, 3]. Acute cardiac complications (ACCs) due to excess amount of CA, including epinephrine, norepinephrine, and sometimes dopamine, in patients with PPGLs may be life-threatening, and timely diagnosis and surgery can be life-saving[1]. To date, only a few retrospective series of PPGLs were reported from cardiac perspectives, revealing the prevalence of ACCs varying from $11-19.3 \%[4-8]$.

ACCs related to PPGLs encompass arrhythmias (bradycardia and tachycardia), Takotsubo-like cardiomyopathy, dilated cardiomyopathy, thromboembolism, acute coronary syndrome, and so on[1]. PPGLs are considered as secondary causes of Takotsubo syndrome (TTS) in the ESC expert consensus, and dozens of cases have been reported[3,9].

In the current study, we retrospectively reviewed the patients diagnosed with PPGLs in our center and screened carefully for patients with ACCs. The prevalence, features and outcomes of PPGLs patients with ACCs were accessed; furthermore, comparisons between patients with and without ACCs were conducted aimed to disclose the patients' features associated with ACCs. Additionally, subclinical myocardial injuries (SMIs) were also investigated to reveal the CAs-induced cardiac damage without causing clinical symptoms.

\section{Methods}

\section{Study populations}

Consecutive patients, diagnosed with PPGLs (clinical or histopathological diagnoses), admitted between January 2013 and July 2020, were included for analysis. Clinical diagnoses were established by classic CAs-related paroxysmal complaints (headache, palpitations, and/or profuse sweating) in combination with adrenal tumors with features suggestive of PPGLs on computed tomography (CT) or magnetic resonance imaging (MRI) scan and elevated plasma CAs or metanephrine (MN) / normetanephrine (NMN) (MNs) at least two times the upper limit of normal (ULN)[10]. Finally, only four patients were clinically diagnosed with PPGLs, and the remaining 185 cases were proven by histopathology.

\section{Clinical assessments}

Data on demographic factors, patterns of hypertension, clinical presentations, electrocardiogram (ECG) alterations, biochemical examinations, pre-operative plasma CAs and MNs levels, echocardiography results, characteristics of tumors, and in-hospital outcomes were recorded. The patterns of hypertension involved normal, sustained (just as essential hypertension), paroxysmal (paroxysms of hypertension on a background of normal blood pressure), mixed (paroxysms of hypertension on a background of sustained hypertension) and unknown pattern[2].

Page 2/13 
Hypertension was based on the diagnosis at admission and pre-operative blood pressure readings, as blood pressure might drop after surgery. We categorized the patterns of tumor secretion into being epinephrine predominant ( $\geq 2 \times$ ULN, including MN), norepinephrine predominant ( $\geq$ $2 \times$ ULN, including NMN), and a combination of both[4]. Maximal tumor diameters were comprehensively determined by gross pathological specimens and pre-operative CT/MRI. The estimated glomerular filtration rate (eGFR) was calculated using CKD-EPI method[11].

The diagnoses of ACCs were established in the presence of at least one of the following clinical situations before surgery: 1) Takotsubo-like cardiomyopathy diagnosed by the following combination[9]: I) transient left ventricular dysfunction with wall-motion abnormality extending beyond a single epicardial coronary artery distribution; II) absence of significant coronary artery stenosis confirmed by coronary artery angiography (CAG) or coronary computed tomography angiography (CTA), or without risk factors and history of angina; III) ECG abnormalities or elevated cardiac biomarkers; IV) no evidence of viral infection; 2) heart failure with unknown etiologies, which was most likely attributed to a CAs release from PPGLs, requiring hospitalizations or intravenous diuretic therapy; 3 ) thromboembolism events most likely of cardiac origin, arrhythmias requiring hospitalizations or antiarrhythmic therapy, and any other cardiac events which were believed to be related with PPGLs.

In addition, SMIs were assessed to reveal detectable myocardial insults before surgery without heart-related symptoms. SMls were defined as any identified abnormality in myocardial injury biomarkers (high-sensitivity cardiac troponin T), N-terminal pro-B type natriuretic peptide (NTproBNP), and systolic dysfunction using two-dimensional (2D) strain analysis in the absence of overloading or other conditions which could alternatively explain the abnormal findings.

In suitable patients, 2D strain analysis was offline performed by a single experienced examiner (Y.X. Miu) using dedicated software (EchoPAC, Norway) and data on parameters including global longitudinal strain (GLS), impaired patterns of longitudinal strain (LS) (e.g., apical, apical sparing which means midventricular or basal strain impairment, segmental, and global) and any other valuable information were documented.

All patient records were anonymized before analysis and the Institutional Review Boards (IRB) waived the requirement for informed consent due to the retrospective nature of the study. The study protocol was approved by the IRB of Soochow University and followed the principles of the Helsinki Declaration.

\section{Statistical analysis}

Continuous variables were accessed for normality by Skewness/kurtosis test and expressed as mean \pm standard deviation (SD) or median (25th, 74th percentage), as appropriate. Means were compared using 2-tailed Student t-test or, in the case of nonnormality, Ranksum test. Categorical variables were shown as numbers (percentages) and differences were detected using Pearson chi-square test or Fisher's exact test, where appropriate. All analyses were carried out using STATA version 14.0 (Stata Corp, College Station, TX). Two-tailed $P$ value less than 0.05 was considered statistically significant.

\section{Results}

As shown in Table 1, a total of 189 cases were included for analysis, among whom median age was $53(38,62)$ years and of whom $52.9 \%$ (100/189) were female. Surprisingly, PPGLs were incidentally found in 59.3\% (112/189) of patients, among whom no symptoms occurred or, in symptomatic cases, symptoms were not considered to be related to space-occupying effect of tumors or CAs-related effect (data not shown). Hypertension occurred in 97 (51.3\%) subjects, and sustained pattern (52/97, 53.6\%) dominated over paroxysmal (15/97, 15.5\%), mixed (19/97, $19.6 \%)$, and unknown $(11 / 97,11.3 \%)$ pattern. With regard to symptoms documented in medical records, abdominal discomfort (nausea or vomiting), chest pain, dyspnea and classic triad symptoms (headache, palpitation, and/or profuse sweating) appeared in $9.5 \%$ (18/189), 3.2\% (6/189), 14.8\% (28/189) and 8.5\% (16/189) of included subjects, respectively. Troponin (55/189, 29.1\%) and NT-proBNP (51/189, 27.0\%) were tested in only approximately one-quarter of study subjects, perhaps due to that only patients suspicious of cardiac damage were screened. 70 (37.0\%) patients were screened for CAs secretion of tumors, as plasma CAs/MNs test was unavailable until January 2018 in our center; however, urine vanillylmandelic acid test was performed in 103 (54.5\%) cases, resulting in 135 (71.4\%) patients undergoing at least one of the aforementioned endocrine tests. The majority of patients received urine routine examination (185/189, 97.9\%), ECG examination (182/189, $96.3 \%)$, blood count examination (187/189, $98.9 \%)$, and renal function examination $(188 / 189,99.5 \%)$. Of note, invasive behavior and the presence of hemorrhage/necrosis on pathology reports were described in $29.7 \%$ and $20.0 \%$ of subjects, respectively. 
Table 1

Clinical profiles of patients diagnosed with PPGLs and comparisons between ACCs group and non-ACCs group.

\begin{tabular}{|c|c|c|c|c|}
\hline & $\begin{array}{l}\text { All } \\
(n=189)\end{array}$ & $\begin{array}{l}\text { non-ACCs } \\
(n=175)\end{array}$ & $\begin{array}{l}\text { ACCs } \\
(n=14)\end{array}$ & $P$ value \\
\hline Male & $89(47.1)$ & $79(45.1)$ & $10(71.4)$ & 0.058 \\
\hline Age (years) & $53(38,62)$ & $54(38,62)$ & $48(38,52)$ & 0.320 \\
\hline History of hypertension & $97(51.3)$ & $86(49.1)$ & $11(78.6)$ & 0.034 \\
\hline \multicolumn{4}{|l|}{ Patterns of hypertension $(n=97)$} & \multirow[t]{4}{*}{0.266} \\
\hline sustained & $52(53.6)$ & $49(57.0)$ & $3(27.3)$ & \\
\hline paroxysmal & $15(15.5)$ & $13(15.1)$ & $2(18.2)$ & \\
\hline mixed & $19(19.6)$ & $15(17.4)$ & $4(36.4)$ & \\
\hline unknown & $11(11.3)$ & $9(10.5)$ & $2(18.2)$ & \\
\hline \multicolumn{5}{|l|}{ Symptoms } \\
\hline nausea or vomiting & $18(9.5 \%)$ & $11(6.3)$ & $7(50.0)$ & $<0.001$ \\
\hline presence of chest pain & $6(3.2)$ & $4(2.3)$ & $2(14.3)$ & 0.065 \\
\hline presence of dyspnea & $28(14.8)$ & $14(8.0)$ & $14(100)$ & $<0.001$ \\
\hline presence of triad ${ }^{\&}$ & $16(8.5)$ & $15(8.6)$ & $1(7.1)$ & 1.000 \\
\hline \multicolumn{5}{|l|}{ ECG changes $(n=182)$} \\
\hline ST-segment changes & $19(10.4)$ & $15(8.9)$ & $4(28.6)$ & 0.043 \\
\hline T wave inversion & $29(15.3)$ & $22(13.1)$ & $7(50.0)$ & 0.002 \\
\hline \multicolumn{5}{|l|}{ Blood parameters } \\
\hline hemoglobin $(\mathrm{g} / \mathrm{L})(\mathrm{n}=187)$ & $132.8 \pm 20.7$ & $132.5 \pm 20.2$ & $136.8 \pm 26.4$ & 0.452 \\
\hline platelet count $\left(\times 10^{9} / L\right)(n=187)$ & $256.1 \pm 81.5$ & $251.9 \pm 76.6$ & 308. $6 \pm 118.5$ & 0.100 \\
\hline white blood cell count $\left(\times 10^{9} / L\right)(n=187)$ & $6.6(5.5,8.6)$ & $6.4(5.4,8.1)$ & $15.6(12.1,18.9)$ & $<0.001$ \\
\hline eGFR $\left(\mathrm{ml} / \mathrm{min} / 1.73 \mathrm{~m}^{2}\right)(\mathrm{n}=188)$ & $108.0(96.9,117.8)$ & $108.0(97.4,117.8)$ & $106.1(49.3,117.3)$ & 0.408 \\
\hline Positive urine protein $(n=185)$ & $24(13.0)$ & $19(11.1)$ & $5(35.7)$ & 0.026 \\
\hline \multicolumn{5}{|l|}{ Cardiac biomarkers } \\
\hline elevation of troponin $(n=55)$ & $19(34.5)$ & $6(14.3)$ & $13(100)$ & $<0.001$ \\
\hline elevation of NT-proBNP* $(n=51)$ & $20(39.2)$ & $7(18.4)$ & $13(100)$ & $<0.001$ \\
\hline \multicolumn{5}{|l|}{ Patterns of secretion $(n=70)$} \\
\hline unknown & $4(5.7)$ & $4(6.8)$ & $0(0)$ & \\
\hline without endocrine & $12(17.1)$ & $12(20.3)$ & $0(0)$ & 0.044 \\
\hline epinephrine predominant & $5(7.1)$ & $4(6.8)$ & $1(9.1)$ & \\
\hline norepinephrine predominant & $25(35.7)$ & $23(39.0)$ & $2(18.2)$ & \\
\hline \multicolumn{5}{|c|}{$\begin{array}{l}\text { Continuous variables are expressed as mean } \pm \text { standard deviation or median ( } 25 \text { th, } 75 \text { th percentage); categorical variables are presented as } \\
\text { number and percentages in parentheses. Missing data varied by variables. }\end{array}$} \\
\hline \multicolumn{5}{|c|}{$\begin{array}{l}\text { Abbreviations: ACCs: acute cardiac complications; cm: centimeter; ECG: electrocardiography; eGFR: estimated glomerular filtration rate; NT- } \\
\text { proBNP: N-terminal pro-B type natriuretic peptide. }\end{array}$} \\
\hline \multicolumn{5}{|c|}{$\&_{\text {defined as presentation of headache, palpitation, and/or profuse sweating. }}$} \\
\hline
\end{tabular}




\begin{tabular}{|c|c|c|c|c|}
\hline & $\begin{array}{l}\text { All } \\
(n=189)\end{array}$ & $\begin{array}{l}\text { non-ACCs } \\
(n=175)\end{array}$ & $\begin{array}{l}\text { ACCs } \\
(n=14)\end{array}$ & $P$ value \\
\hline a combination of both & $24(34.3)$ & $16(27.1)$ & $8(72.7)$ & \\
\hline \multicolumn{5}{|l|}{ Patterns of secretion $(n=70)$} \\
\hline epinephrine secretion & $29(41.4)$ & $20(33.9)$ & $9(81.8)$ & 0.006 \\
\hline \multicolumn{5}{|l|}{ Tumor characteristics } \\
\hline paragangliomas & $52(27.5)$ & $50(28.6)$ & $2(14.3)$ & 0.357 \\
\hline bilateral adrenal tumors $(n=137)$ & $6(4.4)$ & $5(4.0)$ & $1(8.3)$ & 0.429 \\
\hline right adrenal tumors $(n=137)$ & $70(51.1)$ & $64(51.2)$ & $6(50.0)$ & 0.937 \\
\hline maximal tumor diameters $(\mathrm{cm})$ & $4.5(3.2,6)$ & $4.5(3,6)$ & $5.5(4.7,6.5)$ & 0.053 \\
\hline invasive behavior at histology $(n=185)$ & $55(29.7)$ & $47(27.3)$ & $8(61.5)$ & 0.022 \\
\hline hemorrhage/necrosis at histology $(n=185)$ & $37(20.0)$ & $30(17.4)$ & $7(53.9)$ & 0.005 \\
\hline \multicolumn{5}{|c|}{$\begin{array}{l}\text { Continuous variables are expressed as mean } \pm \text { standard deviation or median ( } 25 \text { th, } 75 \text { th percentage); categorical variables are presented as } \\
\text { number and percentages in parentheses. Missing data varied by variables. }\end{array}$} \\
\hline \multicolumn{5}{|c|}{$\begin{array}{l}\text { Abbreviations: ACCs: acute cardiac complications; cm: centimeter; ECG: electrocardiography; eGFR: estimated glomerular filtration rate; NT- } \\
\text { proBNP: N-terminal pro-B type natriuretic peptide. }\end{array}$} \\
\hline \multicolumn{5}{|c|}{${ }^{\&}$ defined as presentation of headache, palpitation, and/or profuse sweating. } \\
\hline
\end{tabular}

\section{Acute cardiac complications}

As depicted in Table 2, fourteen (7.4\%) patients suffered ACCs, including nine cases (4.8\%) with Takotsubo-like cardiomyopathy, three cases (1.6\%) with heart failure with preserved ejection fraction (HFpEF) and one case $(0.5 \%)$ with catecholamine-induced cardiomyopathy. Two patients displayed recurrent episodes of Takotsubo-like cardiomyopathy, of whom one (case 10, two times) with 2 years apart and one (case 9 , three times) with 4 years apart. Predisposing factors were identified in only six (42.9\%) cases, consisting of physical activity or defecation in three cases, operation in one, pregnancy in one and respiratory tract infection in the remaining one. In addition, labile blood pressure was detected in five patients (35.7\%). As for the location of the tumors, diagnoses of PGLs were made in only two cases. In the eleven patients who were screened for plasma catecholamine levels, the pattern of a combination of both $(8 / 11,72.7 \%)$ dominated over the pattern of epinephrine predominance $(1 / 11,9.1 \%)$ and norepinephrine predominance $(2 / 11,18.2 \%)$. All thirteen patients with biomarkers for cardiac injuries displayed elevated troponin T (median $437.6 \mathrm{pg} / \mathrm{ml}$ ) and NT-proBNP (median $8946 \mathrm{pg} / \mathrm{ml}$ ). The mean of left ventricular ejection fraction (LVEF) in acute phase was 0.45 , with a range of $0.21-0.65$; notably, the LVEF reached the normal range by $6-30$ days in all nine patients diagnosed with Takotsubo-like cardiomyopathy. Due to the familial PHEOs and other endocrine glands involvement, case 4 was diagnosed with multiple endocrine neoplasia type 2 without genetic testing. Case 14 was a mid-aged male patient and underwent re-echocardiography examination at 10 months after surgery, which showed no recovery of LVEF, leading to the diagnosis of catecholamine-induced cardiomyopathy. 
Table 2

Characteristics of patients with ACCs.

\begin{tabular}{|c|c|c|c|c|c|c|c|c|c|c|}
\hline \multirow[t]{2}{*}{ Case } & \multirow[t]{2}{*}{$\begin{array}{l}\text { Sex/age } \\
\text { (years) }\end{array}$} & \multirow[t]{2}{*}{$\begin{array}{l}\text { Initial } \\
\text { symptoms }\end{array}$} & \multirow[t]{2}{*}{ Triggers } & \multirow{2}{*}{$\begin{array}{l}\text { Labile } \\
\text { blood } \\
\text { pressure }\end{array}$} & \multirow[t]{2}{*}{$\begin{array}{l}\text { Location of } \\
\text { tumors }\end{array}$} & \multicolumn{2}{|c|}{$\begin{array}{l}\text { Plasma } \\
\text { catecholamine ( } \times \\
\text { ULN) }\end{array}$} & \multirow[t]{2}{*}{$\begin{array}{l}\text { Troponin } \\
(\mathrm{pg} / \mathrm{ml})\end{array}$} & \multirow{2}{*}{$\begin{array}{l}\text { NT- } \\
\text { proBNP } \\
(\mathrm{pg} / \mathrm{ml})\end{array}$} & \multirow[t]{2}{*}{ ECG } \\
\hline & & & & & & $\mathrm{E} / \mathrm{MN}$ & NE/NMN & & & \\
\hline 1 & $51 /$ male & $\begin{array}{l}\text { nausea, } \\
\text { vomiting, } \\
\text { dyspnea, } \\
\text { palpitation }\end{array}$ & $\begin{array}{l}\text { physical } \\
\text { activity }\end{array}$ & no & $\begin{array}{l}\text { left adrenal } \\
\text { PHEO }\end{array}$ & 5.9 & 2.5 & 1159 & 35000 & $\begin{array}{l}\text { sinus } \\
\text { tachycardia, } \\
\text { ST } \\
\text { depression } \\
\text { and T } \\
\text { inversion in } \\
\mathrm{V}_{4-6}\end{array}$ \\
\hline 2 & $38 /$ male & $\begin{array}{l}\text { nausea, } \\
\text { vomiting, } \\
\text { dyspnea, } \\
\text { palpitation, } \\
\text { headache }\end{array}$ & $\begin{array}{l}\text { physical } \\
\text { activity }\end{array}$ & no & $\begin{array}{l}\text { bilateral } \\
\text { adrenal PHEO }\end{array}$ & 15.8 & 4.2 & 1220 & 5976 & $\begin{array}{l}\text { ST } \\
\text { depression } \\
\text { and } \mathrm{T} \\
\text { inversion in } \\
\mathrm{V}_{1-6} \\
\text { prolonged } \\
\text { QTc interval }\end{array}$ \\
\hline 3 & 16/female & dyspnea & infection & no & $\begin{array}{l}\text { right adrenal } \\
\text { PHEO }\end{array}$ & normal & $>12.1$ & 19.3 & 2985 & $\begin{array}{l}\text { sinus } \\
\text { tachycardia }\end{array}$ \\
\hline 4 & $47 /$ male & $\begin{array}{l}\text { dyspnea, } \\
\text { palpitation, } \\
\text { sweating }\end{array}$ & no & yes & $\begin{array}{l}\text { right adrenal } \\
\text { PHEO }\end{array}$ & 4.0 & normal & 2773 & 25492 & $\begin{array}{l}\text { sinus } \\
\text { tachycardia, } \\
\text { nonspecific } \\
\text { T wave } \\
\text { changes }\end{array}$ \\
\hline 5 & 49/female & dyspnea & no & no & $\begin{array}{l}\text { left adrenal } \\
\text { PHEO }\end{array}$ & 11.2 & 11.9 & 77.3 & 4740 & $\begin{array}{l}\text { T wave } \\
\text { inversion in } \\
\text { inferior } \\
\text { leads }\end{array}$ \\
\hline 6 & $62 /$ male & $\begin{array}{l}\text { dyspnea, } \\
\text { palpitation, } \\
\text { sweating }\end{array}$ & no & no & $\begin{array}{l}\text { left adrenal } \\
\text { PHEO }\end{array}$ & 2.6 & 57.5 & 84.3 & 7976 & $\begin{array}{l}\text { diminished } \\
R \text { wave in } \\
V_{2-3}\end{array}$ \\
\hline 7 & $51 /$ male & $\begin{array}{l}\text { dyspnea, } \\
\text { chest pain }\end{array}$ & operation & no & $\begin{array}{l}\text { right adrenal } \\
\text { PHEO }\end{array}$ & - & - & 130.2 & 2250 & $\begin{array}{l}\text { premature } \\
\text { atrial } \\
\text { complex }\end{array}$ \\
\hline 8 & 66/female & $\begin{array}{l}\text { nausea, } \\
\text { vomiting, } \\
\text { dyspnea, } \\
\text { sweating }\end{array}$ & defecation & yes & $\begin{array}{l}\text { right adrenal } \\
\text { PHEO }\end{array}$ & - & - & 437.6 & 33914 & $\begin{array}{l}\text { T wave } \\
\text { inversion in } \\
\text { inferior } \\
\text { leads and } \\
\mathrm{V}_{2-6}\end{array}$ \\
\hline 9 & 29/male & $\begin{array}{l}\text { nausea, } \\
\text { dyspnea, } \\
\text { palpitation, } \\
\text { sweating }\end{array}$ & no & yes & $\begin{array}{l}\text { left adrenal } \\
\text { PHEO }\end{array}$ & 8.4 & 11.4 & 663.6 & 26508 & $\begin{array}{l}\text { sinus } \\
\text { tachycardia, } \\
\text { second } \\
\text { degree AVB }\end{array}$ \\
\hline 10 & 76/male & $\begin{array}{l}\text { nausea, } \\
\text { vomiting, } \\
\text { dyspnea }\end{array}$ & no & no & $\begin{array}{l}\text { right adrenal } \\
\text { PHEO }\end{array}$ & 9.4 & 2.9 & 2178 & $\begin{array}{l}> \\
35000\end{array}$ & $\begin{array}{l}\text { sinus } \\
\text { tachycardia, } \\
\text { low voltage } \\
\text { in limb } \\
\text { leads, ST } \\
\text { depression } \\
\text { in } \mathrm{V}_{3-6}\end{array}$ \\
\hline 11 & 35/female & $\begin{array}{l}\text { dyspnea, } \\
\text { palpitation, } \\
\text { sweating }\end{array}$ & pregnancy & yes & $\begin{array}{l}\text { retroperitoneal } \\
\text { PGL }\end{array}$ & - & - & - & - & $\begin{array}{l}\text { T wave } \\
\text { inversion in } \\
\text { inferior } \\
\text { leads and } \\
\mathrm{V}_{3-6}\end{array}$ \\
\hline 12 & 46/male & $\begin{array}{l}\text { nausea, } \\
\text { vomiting, } \\
\text { dyspnea, } \\
\text { headache }\end{array}$ & no & yes & $\begin{array}{l}\text { right adrenal } \\
\text { PHEO }\end{array}$ & 25.1 & 16.0 & 121.9 & 8946 & $\begin{array}{l}\text { T wave } \\
\text { inversion in } \\
\text { l, aVL, and } \\
\text { V6 }\end{array}$ \\
\hline
\end{tabular}




\begin{tabular}{|c|c|c|c|c|c|c|c|c|c|c|}
\hline \multirow[t]{2}{*}{ Case } & \multirow[t]{2}{*}{$\begin{array}{l}\text { Sex/age } \\
\text { (years) }\end{array}$} & \multirow[t]{2}{*}{$\begin{array}{l}\text { Initial } \\
\text { symptoms }\end{array}$} & \multirow[t]{2}{*}{ Triggers } & \multirow{2}{*}{$\begin{array}{l}\text { Labile } \\
\text { blood } \\
\text { pressure }\end{array}$} & \multirow[t]{2}{*}{$\begin{array}{l}\text { Location of } \\
\text { tumors }\end{array}$} & \multicolumn{2}{|c|}{$\begin{array}{l}\text { Plasma } \\
\text { catecholamine ( } \times \\
\text { ULN) }\end{array}$} & \multirow[t]{2}{*}{$\begin{array}{l}\text { Troponin } \\
(\mathrm{pg} / \mathrm{ml})\end{array}$} & \multirow{2}{*}{$\begin{array}{l}\text { NT- } \\
\text { proBNP } \\
(\mathrm{pg} / \mathrm{ml})\end{array}$} & \multirow[t]{2}{*}{ ECG } \\
\hline & & & & & & $\mathrm{E} / \mathrm{MN}$ & NE/NMN & & & \\
\hline 13 & $42 /$ male & $\begin{array}{l}\text { nausea, } \\
\text { vomiting, } \\
\text { dyspnea, } \\
\text { chest pain, } \\
\text { headache }\end{array}$ & no & no & $\begin{array}{l}\text { left adrenal } \\
\text { PHEO }\end{array}$ & 10.4 & 7.6 & 954.2 & 12913 & $\begin{array}{l}\text { ST } \\
\text { depression } \\
\text { in inferior } \\
\text { leads and } \\
\mathrm{V}_{4-6}, \mathrm{ST} \\
\text { elevation in } \\
\text { aVR }\end{array}$ \\
\hline 14 & $52 /$ male & dyspnea & no & no & pelvic PGL & normal & $>12.1$ & 72.1 & 1208 & $\begin{array}{l}\text { sinus } \\
\text { tachycardia, } \\
\text { LBBB }\end{array}$ \\
\hline
\end{tabular}

Table 2

Characteristics of patients with ACCs (continued).

\begin{tabular}{|c|c|c|c|c|c|c|c|}
\hline Case & $\begin{array}{l}\text { Acute } \\
\text { LVEF (\%) }\end{array}$ & $\begin{array}{l}\text { Strain } \\
\text { analysis }\end{array}$ & $\begin{array}{l}\text { Impaired } \\
\text { pattems in LS }\end{array}$ & $\begin{array}{l}\text { Acute } \\
\text { global LS }\end{array}$ & $\begin{array}{l}\text { Coronary } \\
\text { artery evaluation }\end{array}$ & $\begin{array}{l}\text { Recovery } \\
\text { on imaging }\end{array}$ & Clinical diagnosis \\
\hline 1 & 0.38 & yes & $\begin{array}{l}\text { apical } \\
\text { ballooning }\end{array}$ & -5.5 & CTA: $(-)$ & 6 days & Takotsubo syndrome \\
\hline 2 & 0.39 & yes & apical sparing & -10.7 & CAG: (-) & 7 days & Takotsubo syndrome \\
\hline 3 & 0.45 & yes & apical sparing & -4.8 & $\begin{array}{l}\text { without risk } \\
\text { factors }\end{array}$ & 30 days & Takotsubo syndrome \\
\hline 4 & 0.21 & yes & apical sparing & -6.1 & $\begin{array}{l}\text { without risk } \\
\text { factors }\end{array}$ & 14 days & Takotsubo syndrome \\
\hline 5 & 0.62 & no & - & - & CAG: $(-)$ & - & HFpEF \\
\hline 6 & 0.64 & yes & apical sparing & -16.8 & CTA: $(-)$ & - & HFpEF \\
\hline 7 & 0.43 & no & - & - & $\begin{array}{l}\text { CTA: p-RCA } 50 \% \\
\text { stenosis }\end{array}$ & 12 days & Takotsubo syndrome \\
\hline 8 & 0.61 & no & - & - & no & - & HFpEF, ischemic stroke \\
\hline 9 & 0.36 & no & - & - & $\begin{array}{l}\text { without risk } \\
\text { factors }\end{array}$ & 11 days & $\begin{array}{l}\text { Recurrent Takotsubo } \\
\text { syndrome ( } 3 \text { times) }\end{array}$ \\
\hline 10 & 0.38 & yes & segmental & -21.2 & CTA: (-) & 11 days & $\begin{array}{l}\text { Recurrent Takotsubo } \\
\text { syndrome ( } 2 \text { times) }\end{array}$ \\
\hline 11 & 0.65 & no & - & - & $\begin{array}{l}\text { without risk } \\
\text { factors }\end{array}$ & - & HFpEF \\
\hline 12 & 0.59 & yes & apical sparing & -9.6 & $\begin{array}{l}\text { without risk } \\
\text { factors }\end{array}$ & 7 days & Takotsubo syndrome \\
\hline 13 & 0.35 & yes & apical sparing & -13.6 & CAG: $(-)$ & 15 days & Takotsubo syndrome \\
\hline 14 & 0.28 & yes & global & - & CAG: (-) & $\begin{array}{l}\text { without recovery in } \\
10 \text { months }\end{array}$ & $\begin{array}{l}\text { Catecholamine-induced } \\
\text { cardiomyopathy }\end{array}$ \\
\hline \multicolumn{8}{|c|}{$\begin{array}{l}\text { Abbreviations: AVB: atrioventricular block; CAG: coronary artery angiography; CTA: coronary computed tomography angiography; E: } \\
\text { epinephrine; HFpEF: heart failure with preserved ejection fraction; LBBB: left bundle branch block; LVEF: left ventricular ejection fraction; LS: } \\
\text { longitudinal strain; LV: left ventricle; MN: metanephrine; NE: norepinephrine; NMN: normetanephrine; PGL: paraganglioma; PHEO: } \\
\text { pheochromocytoma; p-RCA: proximal right coronary artery; ULN: upper limit of normal. Other abbreviations as shown in Table 1. }\end{array}$} \\
\hline
\end{tabular}

In comparison to non-ACCs group, ACCs group shared similar characteristics in sex (male, 71.4\% vs $45.1 \%, P=0.058$ ) and age (median, 48 vs $54, P=0.320)$. As expected, more patients with ACCs had hypertension (78.6\% vs $49.1 \%, P=0.034)$; however, the patterns of hypertension didn't differ in two groups $(P=0.266)$. Surprisingly, the proportions of subjects who displayed classic triad symptoms $(7.1 \%$ vs $8.6 \%, P=1.000)$ and chest pain (14.3\% vs $2.3 \%, \mathrm{P}=0.065)$ were similar and quite low among two groups; nevertheless, much more patients showed gastrointestinal symptoms $(50.0 \%$ vs $6.3 \%, \mathrm{P}<0.001)$ and dyspnea $(100 \%$ vs $8.0 \%, \mathrm{P}<0.001)$ in ACCs group. Expectedly, with regard to ECG changes, patients 
with ACCs were more prevalent in both ST-segment changes (28.6\% vs $8.9 \%, P=0.043)$ and $T$ wave inversions $(50.0 \%$ vs $13.1 \%, P=0.002)$ as compared to those without ACCs. In terms of laboratory findings, proteinuria was more likely to be prevalent in ACCs group (35.7\% vs $11.1 \%$, $\mathrm{P}$ = 0.026); likewise, ACCs group had higher median level of white blood cell (median, 15.6 vs 6.4, $\mathrm{P}<0.001$ ). Additionally, as for patterns of secretion, a combination of both dominated in patients with ACCs ( $\mathrm{P}=0.044)$; moreover, a higher prevalence of epinephrine-producing PPGLs (81.8\% vs 33.9\%. P = 0.006) was observed in ACCs group. Overall, the proportions of paragangliomas, bilateral adrenal tumors, and right adrenal tumors were similar between two groups; however, patients with ACCs exhibited a trend for having larger maximal tumor diameters, but the difference didn't reach statistical significance (median, 5.5 vs 4.5, P = 0.053). Interestingly, more patients in ACCs group showed invasive behavior $(61.5 \%$ vs $27.3 \%, P=0.022)$ and hemorrhage/necrosis $(53.9 \%$ vs $17.4 \%, P=0.005)$ on pathology report as compared with patients in non-ACCs group.

\section{Strain analysis}

As shown in Table 2, 2D strain analysis was carried out in 9 (64.3\%) out of 14 patients with ACCs, and technical reasons accounted for the failure of analysis in the rest 5 cases. Strikingly, 6 (66.7\%) patients displayed the pattern of relative apical sparing in LS; meanwhile, of the rest 3 patients, one (case 1) showed the pattern of apical ballooning, one (case 10) displayed the pattern of segmental dysfunction, and the last one (case 14) showed the pattern of global dysfunction. Of 7 patients with Takotsubo-like cardiomyopathy who underwent strain analysis, one patient (case 1) (14.3\%) exhibited the typical apical ballooning pattern; however, 5 (71.4\%) (case 2, 3, 4, 12, 13) cases showed the pattern of relative apical sparing in LS. Visual examples of patterns of 'apical ballooning', 'apical sparing', 'global' and 'segmental' in LS are depicted in Fig. 1 (panel A-G). Finally, the mean value of GLS was $-11.0 \pm 5.8$, with a range of -5.5 to -21.2 .

\section{Subclinical myocardial injuries}

The prevalence of SMIs was potential to be underestimated owing to unthorough investigations or to be an overestimation because of the prescription of examinations being at discretion of physicians in this study. Only 74 (39.2\%) patients had echocardiography examination, 55 (29.1\%) received troponin test and 51 (27.0\%) underwent NT-proBNP test; then, only 91 (48.2\%) patients received at least one of the aforementioned three examinations. After excluding patients with ACCs, 21 (27.2\%) cases out of 77 experienced SMls, including elevated NTproBNP in 15, elevated troponin in 4 and abnormal findings on echocardiography examinations in 10 patients (shown in Table 3 ). 
Table 3

Characteristics of patients with SMIs.

\begin{tabular}{|c|c|c|c|c|c|c|c|c|}
\hline \multirow[t]{2}{*}{ Case } & \multirow[t]{2}{*}{$\begin{array}{l}\text { Sex/age } \\
\text { (years) }\end{array}$} & \multirow[t]{2}{*}{$\begin{array}{l}\text { Location of } \\
\text { tumors }\end{array}$} & \multicolumn{2}{|c|}{$\begin{array}{l}\text { Blood } \\
\text { catecholamine (x } \\
\text { ULN) }\end{array}$} & \multirow[t]{2}{*}{$\begin{array}{l}\text { Troponin } \\
\text { (pg/ml) }\end{array}$} & \multirow{2}{*}{$\begin{array}{l}\text { NT- } \\
\text { proBNP } \\
(\mathrm{pg} / \mathrm{ml})\end{array}$} & \multirow[t]{2}{*}{ Echocardiography } & \multirow[t]{2}{*}{$\begin{array}{l}\text { Impaired patterns on } \\
\text { strain analysis* }\end{array}$} \\
\hline & & & $\mathrm{E} / \mathrm{MN}$ & NE/NMN & & & & \\
\hline 1 & $55 /$ male & $\begin{array}{l}\text { retroperitoneal } \\
\text { PGL }\end{array}$ & normal & 15.3 & normal & normal & $\begin{array}{l}\text { enlarged LA and LV, } \\
\text { LVH }\end{array}$ & $\begin{array}{l}\text { anterior and lateral } \\
\text { segments }\end{array}$ \\
\hline 2 & 35/female & $\begin{array}{l}\text { right adrenal } \\
\text { PHEO }\end{array}$ & normal & 33.1 & - & - & LVH & apical sparing \\
\hline 3 & $31 /$ male & $\begin{array}{l}\text { retroperitoneal } \\
\text { PGL }\end{array}$ & - & - & 21.4 & 216.4 & LVH & - \\
\hline 4 & $56 /$ male & $\begin{array}{l}\text { retroperitoneal } \\
\text { PGL }\end{array}$ & normal & 12.2 & 337.4 & 137.2 & normal & normal \\
\hline 5 & 47/female & $\begin{array}{l}\text { left adrenal } \\
\text { PHEO }\end{array}$ & - & - & normal & 350 & enlarged LA & - \\
\hline 6 & 58/female & $\begin{array}{l}\text { left adrenal } \\
\text { PHEO }\end{array}$ & 9.1 & 2.7 & normal & 366.6 & normal & apical sparing \\
\hline 7 & $38 /$ male & $\begin{array}{l}\text { left adrenal } \\
\text { PHEO }\end{array}$ & normal & $>12.1$ & normal & 164.2 & enlarged LA, LVH & $\begin{array}{l}\text { anterior and lateral } \\
\text { segments }\end{array}$ \\
\hline 8 & $65 /$ male & $\begin{array}{l}\text { retroperitoneal } \\
\text { PGL }\end{array}$ & - & - & 21 & 2140 & - & - \\
\hline 9 & 70/male & $\begin{array}{l}\text { left adrenal } \\
\text { PHEO }\end{array}$ & 4.9 & 4.8 & - & - & normal & basal inferior segment \\
\hline 10 & 54/female & $\begin{array}{l}\text { right adrenal } \\
\text { PHEO }\end{array}$ & - & - & normal & 153 & normal & $\begin{array}{l}\text { anterior and lateral } \\
\text { segments }\end{array}$ \\
\hline 11 & 54/female & $\begin{array}{l}\text { right adrenal } \\
\text { PHEO }\end{array}$ & 38.9 & 12.1 & normal & 778.4 & LVH & - \\
\hline 12 & 67/female & $\begin{array}{l}\text { left adrenal } \\
\text { PHEO }\end{array}$ & normal & 5.2 & - & - & enlarged LA, LVH & $\begin{array}{l}\text { anterior and lateral } \\
\text { segments }\end{array}$ \\
\hline 13 & $31 /$ male & $\begin{array}{l}\text { left adrenal } \\
\text { PHEO }\end{array}$ & normal & 8.4 & normal & 303.8 & - & - \\
\hline 14 & 39/female & $\begin{array}{l}\text { left adrenal } \\
\text { PHEO }\end{array}$ & 7.6 & 9.9 & normal & 1008 & normal & \\
\hline 15 & $67 /$ male & $\begin{array}{l}\text { right adrenal } \\
\text { PHEO }\end{array}$ & 2.8 & 7.4 & - & - & $\begin{array}{l}\text { segmental dysfunction, } \\
\text { enlarged LA }\end{array}$ & $\begin{array}{l}\text { anterior and lateral } \\
\text { segments }\end{array}$ \\
\hline 16 & $56 /$ female & $\begin{array}{l}\text { right adrenal } \\
\text { PHEO }\end{array}$ & 4.0 & 1.9 & normal & 322.6 & - & - \\
\hline 17 & 55/female & $\begin{array}{l}\text { right adrenal } \\
\text { PHEO }\end{array}$ & normal & normal & - & - & $\begin{array}{l}\text { pulmonary } \\
\text { hypertension }\end{array}$ & anterior segment \\
\hline 18 & 35/female & $\begin{array}{l}\text { right adrenal } \\
\text { PHEO }\end{array}$ & 4.0 & 4.0 & normal & 408.5 & - & - \\
\hline 19 & 23/male & $\begin{array}{l}\text { right adrenal } \\
\text { PHEO }\end{array}$ & normal & 8.5 & normal & 337.7 & - & - \\
\hline 20 & 57/female & $\begin{array}{l}\text { right adrenal } \\
\text { PHEO }\end{array}$ & normal & normal & 48.39 & 215.8 & - & - \\
\hline 21 & 40/female & $\begin{array}{l}\text { left adrenal } \\
\text { PHEO }\end{array}$ & - & - & normal & 770 & - & - \\
\hline Abbrev & tions: LA: Ie & atrium; LVH: lef & ventricul & hypertroph & Other abbr & jiations as & shown in Tables 1 and 2. & \\
\hline
\end{tabular}


In this retrospective study with a large sample size (189 cases), we demonstrated a relatively high prevalence (7.4\%) of ACCs owing to catecholamine excess in patients with PPGLs. Moreover, 9 out of 14 patients with ACCs displayed Takotsubo-like cardiomyopathy, and 2D strain analysis further revealed that the predominant impaired pattern was apical sparing pattern (5/7), which dominated over the typical apical ballooning pattern seen in TTS (1/7). In fact, we believed that part of, if not all, patients diagnosed with HFpEF had gone through the clinical course of Takotsubo-like cardiomyopathy, since strain analysis showed impaired strain in case 6. Of note, SMls had a fairly high incidence $(27.2 \%)$ in patients who underwent cardiac biomarker examinations or echocardiography examinations, suggesting the universality of CAsrelated cardiac injuries.

In this study, the majority (59.3\%) of PPGLs were incidental findings on abdominal ultrasonogram or CT/MRI scan rather than PPGLs-related symptoms. The main discovery method is consistent with recent cohort studies, implying the changes in PPGLs recognition due to the widespread use of cross-sectional imaging; hence, the relatively low prevalence of hypertension and symptoms might be explained by the fact that most PPGLs were incidentally found in recent years[12, 13]. Nausea or vomiting occurred in half of the patients with ACCs, which should be partly, if not totally, ascribed to excessive CAs secretion[13]. The data was surprising to us given less than ten percent of patients showed classical triad symptoms, which was lower than the percent (17\%) reported by recent study[13] and might be underestimated owing to the retrospective nature of the study. As with the strikingly low prevalence (4\%) of classical triad in patients with PPGLs-induced reversible cardiomyopathy reported by recent review, the low rate in our study could help remind clinicians to consider PPGLs in the context of reversible cardiomyopathy with unclear etiology and not to rely on the classical triad as a diagnostic threshold[3].

Proteinuria was more prevalent in patients with ACCs as compared with non-ACCs controls, implying the more severe kidney injury brought by CAs or cytokines released from PPGLs[14]. Worth mentioning, epinephrine secretion was present in most cases (81.8\%) with ACCs, which was consistent with previous studies[4], indicating episodical release of epinephrine causing sudden cardiac decompensation as opposed to persistent store and release for norepinephrine[15, 16]. A trend for larger tumor diameter was observed in ACCs-group compared to non-ACCs group ( $P=0.053)$, and inconsistent correlations between tumor size and ACCs had been reported in prior studies[5-7]. Presence of hemorrhage/necrosis at histology was more often seen in subjects with ACCs than those without, suggesting the possible role as triggers played by hemorrhage/necrosis[2, 17]. In agreement with the recognition that all PPGLs have a metastatic risk, we reported a fairly high rate of invasive behavior on pathology report (29.7\%). Additionally, invasive behavior seemed to be associated with ACCs; however, the result should be considered exploratory owing to the lack of standardization of interpretation of pathology results and well-accepted histological features indicating metastasis[18].

The prevalence (4.8\%) of Takotsubo-like cardiomyopathy in our cohort was comparable with the $1.4 \sim 5.6 \%$ prevalence reported in previous studies[4-8]. The apical sparing pattern (5/7), indicating mid-ventricular and/or basal impairment, dominated in the impaired patterns of LS whereas prior reviews revealed a predominance (48\%) of classical apical ballooning pattern on routine echocardiography examinations[3, 4]. The different impaired patterns between PPGLs-induced TTS and classic TTS hint that distinct pathogenetic mechanisms may exist among them[19, 20]. Recurrent TTS associated with PPGLs were reported and systematically reviewed by our team recently[21]; note, two patients experienced recurrent episodes of TTS and were diagnosed as myocarditis in $2 \sim 4$ years before identifying PPGLs in this cohort, highlighting the value of screening for the presence of PPGLs among these patients.

Unexpectedly, a quite high prevalence (27.2\%) of SMIs in patients with PPGLs was observed in our study, implying that cardiac injury without clinical manifestations is common in the presence of excessive CAs release. The abnormalities in ECG examinations, including sinus tachycardia, atrial fibrillation, premature complex, and nonspecific ST-T changes, were not deemed as SMls because they were unspecific for PPGLs-related cardiac injuries; hence, the occurrence rate of SMIs might be underestimated.

\section{Strengthens and limitations of this study}

This study is a first study aimed to investigate the PPGLs-induced ACCs and SMIs in East-Asian population with a large sample size. It is the first time to characterize the echocardiography abnormalities on strain analysis and demonstrate that the primary pattern was apical sparing pattern. In addition, the high prevalence, possibly being underestimated, of SMls observed in this study were not reported in previous cohort studies. However, limitations are obvious, including the followings: firstly, we were not able to perform comprehensive assessments of the cardiac injuries attributed to tremendous amount of CAs because of the retrospective nature of the study; secondly, due to lack of genetic screening, the diagnoses made in this cohort failed to meet the requirement in current guidelines[10]; thirdly, the low number of events made it impossible to conduct regression analyses to reveal the independent factors associated with ACCs; finally, it should be cautious to expand the results owing to center-specific referral bias.

\section{Conclusion}


Collectively, this study with retrospective design revealed that one in every fourteen PPGLs patients presented with ACCs. The main impaired pattern in patients with TTS on strain analysis was apical sparing pattern, which is distinct from previous studies. Moreover, nearly one-third of patients without symptoms had SMIs. Clinical clues suggestive of PPGLs should not rely solely on adrenergic symptoms, particularly classic triad, due to its extremely low prevalence. Clinicians should consider PPGLs in patients with acute reversible cardiomyopathy, especially with an apical sparing pattern in LS.

\section{Abbreviations}

ACCs: acute cardiac complications; CAG: coronary artery angiography; CAs: catecholamines; CT: computed tomography; CTA: coronary computed tomography angiography; ECG: electrocardiography; eGFR: estimated glomerular filtration rate; GLS: global longitudinal strain; HFpEF: heart failure with preserved ejection fraction; LVEF: left ventricular ejection fraction; LS: longitudinal strain; MN: metanephrine; MNs: metanephrine / normetanephrine; MRI: magnetic resonance imaging; NMN: normetanephrine; NT-proBNP: N-terminal pro-B type natriuretic peptide. PGLs: paragangliomas; PHEOs: pheochromocytomas; PPGLs: pheochromocytomas and paragangliomas; SD: standard deviation; SMIs: subclinical myocardial injuries; TTS: Takotsubo syndrome; ULN: upper limit of normal.

\section{Declarations}

\section{Ethics approval and consent to participate}

All patient records were anonymized before analysis and the requirement for informed consent was waived by the Institutional Review Boards (IRB) due to the retrospective nature of the study. The study protocol was approved by the IRB of Soochow University and followed the principles of the Helsinki Declaration.

\section{Consent for publication}

Not applicable.

\section{Availability of data and material}

The date used for analysis can be available by corresponding author per reasonable request.

\section{Competing interests}

The author declare that they have no competing interests.

\section{Funding}

No funding was received for this study.

\section{Authors' contributions}

Y-L D designed this study. JZ, J-T H and HX made substantial contributions to acquisition of the data. Y-L D, JZ and HX were involved in data analysis. 2D strain analysis was performed by Y-X M. Y-L D, JZ and HX wrote the manuscript. All author approved the final version of the manuscript.

\section{Acknowledgements}

None.

\section{References}

1. S YH, Falhammar H: Cardiovascular Manifestations and Complications of Pheochromocytomas and Paragangliomas. Journal of clinical medicine 2020, 9(8).

2. Lenders JWM, Eisenhofer G, Mannelli M, Pacak K: Phaeochromocytoma. The Lancet 2005, 366(9486):665-675.

3. Zhang R, Gupta D, Albert SG: Pheochromocytoma as a reversible cause of cardiomyopathy: Analysis and review of the literature. Int $J$ Cardiol 2017, 249:319-323.

4. Gagnon N, Mansour S, Bitton Y, Bourdeau I: TAKOTSUBO-LIKE CARDIOMYOPATHY IN A LARGE COHORT OF PATIENTS WITH PHEOCHROMOCYTOMA AND PARAGANGLIOMA. Endocr Pract 2017, 23(10):1178-1192. 
5. Riester A, Weismann D, Quinkler M, Lichtenauer UD, Sommerey S, Halbritter R, Penning R, Spitzweg C, Schopohl J, Beuschlein F et al: Lifethreatening events in patients with pheochromocytoma. European journal of endocrinology 2015, 173(6):757-764.

6. Giavarini A, Chedid A, Bobrie G, Plouin PF, Hagège A, Amar L: Acute catecholamine cardiomyopathy in patients with phaeochromocytoma or functional paraganglioma. Heart (British Cardiac Society) 2013, 99(19):1438-1444.

7. Zelinka T, Petrák O, Turková H, Holaj R, Strauch B, Kršek M, Vránková AB, Musil Z, Dušková J, Kubinyi J et al: High incidence of cardiovascular complications in pheochromocytoma. Hormone and metabolic research = Hormon- und Stoffwechselforschung = Hormones et metabolisme 2012, 44(5):379-384.

8. Park JH, Kim KS, Sul JY, Shin SK, Kim JH, Lee JH, Choi SW, Jeong JO, Seong IW: Prevalence and patterns of left ventricular dysfunction in patients with pheochromocytoma. J Cardiovasc Ultrasound 2011, 19(2):76-82.

9. Ghadri JR, Wittstein IS, Prasad A, Sharkey S, Dote K, Akashi YJ, Cammann VL, Crea F, Galiuto L, Desmet W et al: International Expert Consensus Document on Takotsubo Syndrome (Part I): Clinical Characteristics, Diagnostic Criteria, and Pathophysiology. European heart journal 2018, 39(22):2032-2046.

10. Lenders JWM, Kerstens MN, Amar L, Prejbisz A, Robledo M, Taieb D, Pacak K, Crona J, Zelinka T, Mannelli M et al: Genetics, diagnosis, management and future directions of research of phaeochromocytoma and paraganglioma: a position statement and consensus of the Working Group on Endocrine Hypertension of the European Society of Hypertension. Journal of hypertension 2020, 38(8):1443-1456.

11. Levey AS, Stevens LA, Schmid CH, Zhang YL, Castro AF, 3rd, Feldman HI, Kusek JW, Eggers P, Van Lente F, Greene T et al: A new equation to estimate glomerular filtration rate. Annals of internal medicine 2009, 150(9):604-612.

12. Gruber LM, Hartman RP, Thompson GB, McKenzie TJ, Lyden ML, Dy BM, Young WF, Bancos I: Pheochromocytoma Characteristics and Behavior Differ Depending on Method of Discovery. J Clin Endocrinol Metab 2019, 104(5):1386-1393.

13. Falhammar $\mathrm{H}$, Kjellman M, Calissendorff $\mathrm{J}$ : Initial clinical presentation and spectrum of pheochromocytoma: a study of 94 cases from a single center. Endocrine connections 2018, 7(1):186-192.

14. Porzig A, Matthay KK, Dubois S, Pampaloni M, Damon L, Hawkins R, Goldsby R, Hollinger F, Fitzgerald P: Proteinuria in metastatic pheochromocytoma is associated with an increased risk of Acute Respiratory Distress Syndrome, spontaneously or after therapy with 131I-meta-iodobenzylguanidine (131I-MIBG). Hormone and metabolic research = Hormon- und Stoffwechselforschung = Hormones et metabolisme 2012, 44(7):539-542.

15. Gupta G, Pacak K: Precision Medicine: An Update on Genotype/Biochemical Phenotype Relationships in Pheochromocytoma/Paraganglioma Patients. Endocrine Practice 2017, 23(6):690-704.

16. Eisenhofer G, Huynh TT, Elkahloun A, Morris JC, Bratslavsky G, Linehan WM, Zhuang Z, Balgley BM, Lee CS, Mannelli M et al: Differential expression of the regulated catecholamine secretory pathway in different hereditary forms of pheochromocytoma. Am $\mathrm{J}$ Physiol Endocrinol Metab 2008, 295(5):E1223-1233.

17. Prejbisz A, Lenders JW, Eisenhofer G, Januszewicz A: Cardiovascular manifestations of phaeochromocytoma. Journal of hypertension 2011, 29(11):2049-2060.

18. Turchini J, Cheung VKY, Tischler AS, De Krijger RR, Gill AJ: Pathology and genetics of phaeochromocytoma and paraganglioma. Histopathology 2018, 72(1):97-105.

19. S YH: Clinical Features and Outcome of Pheochromocytoma-Induced Takotsubo Syndrome: Analysis of 80 Published Cases. Am J Cardiol 2016, 117(11):1836-1844.

20. Awad HH, McNeal AR, Goyal H: Reverse Takotsubo cardiomyopathy: a comprehensive review. Annals of translational medicine 2018, 6(23):460.

21. Dai YL, Zhou J, Lin J, Hu JT, Zhao X: [Recurrent Takotsubo syndrome associated with pheochromocytoma: a case report]. Zhonghua nei ke za zhi 2020, 59(6):464-467.

\section{Figures}




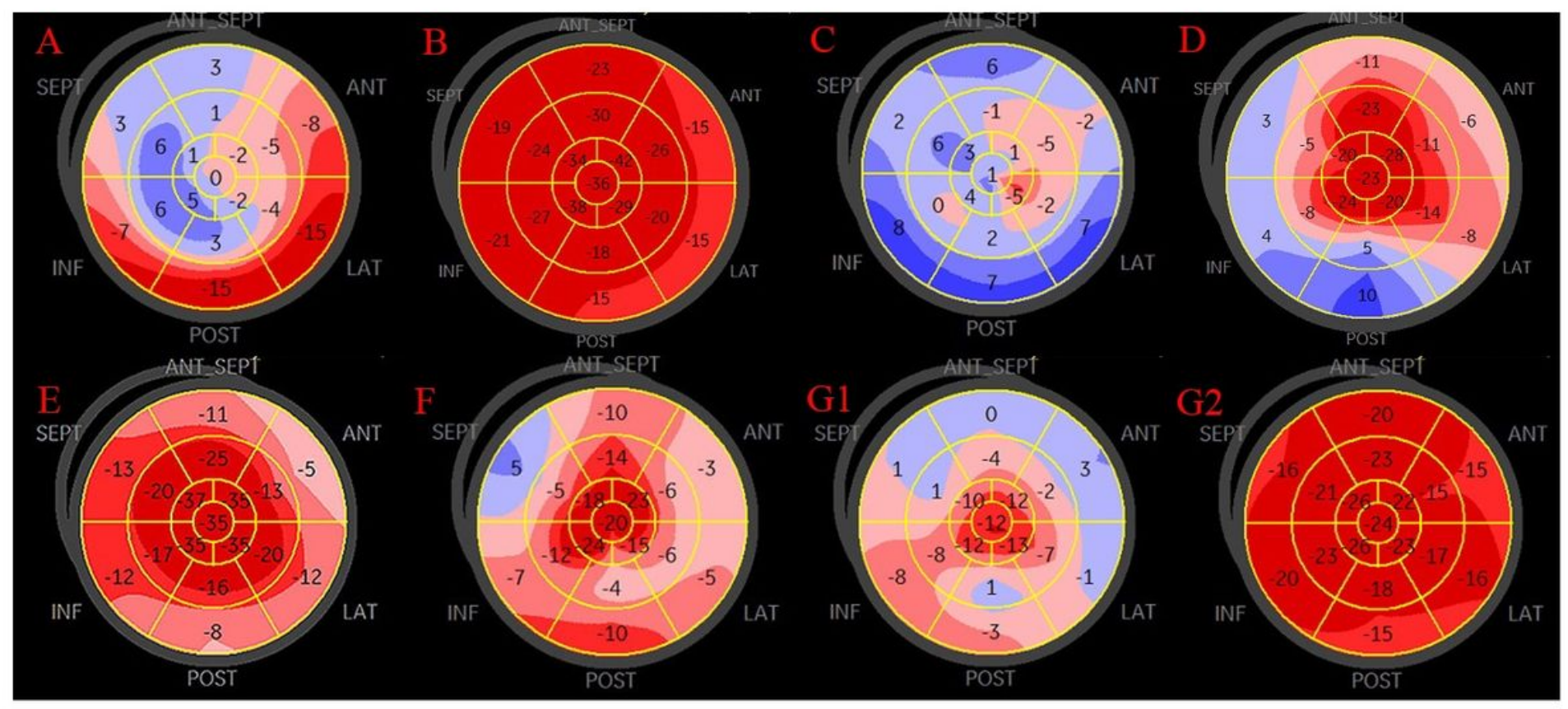

\section{Figure 1}

Representative impaired patterns in longitudinal strain for each subgroup. (A) The pattern of apical ballooning in case 1. (B) The pattern of segmental dysfunction in case 10. (C) The pattern of global dysfunction in case 14. (D-F) The patterns of apical sparing in case 2,6 , and 12. $(G 1,2)$ The apical sparing pattern in acute phase and a nearly full recovery 14 days later in case 4. 